\title{
Expression and potential correlation among Forkhead box protein M1, Caveolin-1 and E-cadherin in colorectal cancer
}

\author{
JING ZHANG ${ }^{*}$, KUNDONG ZHANG ${ }^{*}$, LISHENG ZHOU*, WEIDONG WU, TAO JIANG, \\ JUN CAO, KEJIAN HUANG, ZHENGJUN QIU and CHEN HUANG \\ Department of General Surgery, First People's Hospital Affiliated to Shanghai Jiao Tong University, \\ Shanghai 200080, P.R. China
}

Received May 4, 2015; Accepted June 7, 2016

DOI: $10.3892 / 01.2016 .4915$

\begin{abstract}
The aim of the present study was to investigate the expression and functions of Forkhead box protein M1 (FoxM1), Caveolin-1 (Cav-1) and E-cadherin in colorectal cancer (CRC), and to determine the correlations among these proteins in CRC development and progression. The protein expression of FoxM1, Cav-1 and E-cadherin was identified using a human CRC and normal tissue microarray. A standard immunohistochemistry assay was performed employing anti-FoxM1, anti-Cav-1 and anti-E-cadherin antibodies. The clinicopathological significance of FoxM1, Cav-1 and E-cadherin in CRC was determined, and correlations were investigated between FoxM1 and Cav-1, FoxM1 and E-cadherin, Cav-1 and E-cadherin, respectively. The level of FoxM1, Cav-1 and E-Cadherin protein expression in CRC was found to be associated with pathological grade, tumor clinical stages and the presence of metastasis, respectively. Elevated expression of FoxM1 and Cav-1 was observed in the CRC tissues, and a significant correlation was found between the two proteins in CRC. However, it was also observed that FoxM1 was overexpressed while E-cadherin expression was low, indicating that there was a negative correlation between FoxM1 expression and E-cadherin expression. Moreover, there was also a negative correlation between Cav-1 and E-cadherin expression. Overall, the elevated expression of FoxM1 and Cav-1 in a human CRC microarray provided novel clinical evidence to elucidate the fact that they may play a critical role in the development and progression of CRC by negatively regulating E-cadherin expression. Furthermore,
\end{abstract}

Correspondence to: Dr Chen Huang or Dr Zhengjun Qiu, Department of General Surgery, First People's Hospital Affiliated to Shanghai Jiao Tong University, 100 Haining Road, Shanghai 200080, P.R. China

E-mail: richard-hc@hotmail.com

E-mail: zhengjunqiu283@126.com

${ }^{*}$ Contributed equally

Key words: foxM1, caveolin-1, E-cadherin, colorectal cancer the positive correlation between FoxM1 and Cav-1 suggested that the proteins may constitute a novel signaling pathway in human CRC.

\section{Introduction}

As a pervasive cancer worldwide, colorectal cancer (CRC) is the third most commonly diagnosed cancer and the fourth major cause of cancer-associated mortality, with an estimated 1.2 million new cancer cases and >600,000 mortalities annually (1). CRC in China ranks among the 10 leading cancers in terms of incidence, with an occurrence rate ranking sixth in humans. In 2010, there were 132,110 recorded mortalities due to $\mathrm{CRC}$, with mortality in men and women ranking fifth and sixth among all cancers, respectively (2). Despite improvements in surgical techniques, adjuvant chemotherapy and neoadjuvant radiotherapy, the prognosis of advanced CRC remains unsatisfactory, with a 5-year relative survival rate of only $10 \%$ in stage IV patients (3). Therefore, there is an essential requirement for a $\mathrm{n}$ improved understanding of the biological features and pathogenesis of $\mathrm{CRC}$, which will be useful in the investigation of novel treatments and prognostic markers for this disease.

Forkhead box protein M1 (FoxM1), previously known as MPP-2, FKHL-16 or Trident, belongs to the abundant family of forkhead transcription factors, characterized by an evolutionary conserved winged helix DNA-binding domain termed Forkhead box (4). It has been acknowledged that the expression of FoxM1 is associated with proliferating cells, with levels particularly increasing at the entry to the S-phase of the cell cycle and reaching a peak during the G2 and $\mathrm{M}$ phases, but that it is absent in quiescent and terminally-differentiated cells (5). Furthermore, it is ubiquitously reported that FoxM1 plays a critical role in tumorigenesis, including angiogenesis, invasion and metastasis, and that the elevated expression levels of FoxM1 contribute to a poor prognosis and metastasis in diverse tumors (5). Emerging data has shown that FoxM1 is overexpressed in solid tumors of the bladder, prostate, lungs, ovaries, breasts, liver, kidneys, stomach, pancreas and colon (6). Furthermore, it is not unexpected that the decreased expression of FoxM1 when using FoxM1 short hairpin RNA leads to the reduction of cell proliferation, migration and invasion in human 
tumor cell lines (7). Specifically, the expression of FoxM1 is significantly observed in samples from colon cancer patients compared with lower expression in corresponding adjacent normal tissues. Moreover, the enforced expression level of FoxM1 drastically promotes the proliferation, development and growth of colon tumors in FoxM1 transgenic mice (8). Nevertheless, the underlying molecular regulatory mechanisms of FoxM1 in the promotion of CRC remain unclear, and the roles of FoxM1 in the process of CRC development and progression remain to be elucidated.

Caveolin-1 (Cav-1), a principal structural component of caveolae and a 21- to 24-kDa integral membrane protein acting as a scaffolding protein, is implicated in various cellular events by interacting with diverse molecular complexes, and it may play an irreplaceable role in the transformation and tumorigenesis of cells (9). Notably, Cav-1 is defined with an ambiguous role in cancer. The wealth of data available indicate that the role of Cav-1 as a tumor suppressor or promoter is dependent on the type of tissue; for example, Cav-1 acts as a tumor suppressor in lung cancer, ovarian carcinoma and sarcomas, whereas it act as a tumor promoter in prostate cancer and esophageal squamous cell carcinomas (10). Based on previous studies, compared with the increased expression of Cav-1 in normal gastric tissues, there is a lower expression of Cav-1 in paired gastric adenocarcinoma tissues; the results obtained in gastric carcinoma cell lines are also consistent with these observations, supporting the fact that Cav-1 may be a tumor suppressor in gastric cancer (11). With respect to the expression of Cav-1 in colon cancer, controversy remains. One previous study stated that the expression level of Cav-1 is decreased in human colon cancer, contributing greatly to cellular migration and invasiveness (12). By contrast, other emerging evidence supports the overexpression of Cav-1 in colon cancer in comparison with normal colon tissues (13). Considering all the aforementioned evidence, it is necessary and essential for the expression and role of Cav-1 to be determined in CRC.

E-cadherin, a single-span transmembrane glycoprotein, is indispensable for the function of epithelial adherens junctions through its interactions with adjacent E-cadherin molecules secreted by contiguous cells (14). It has been widely illustrated in different assays that the decreased expression of E-cadherin is implicated in various tumors, such as pancreatic cancer, non-small cell lung cancer and CRC, by acting in the process of epithelial-mesenchymal transition (EMT) (15-17). Kong et al verified that the elevated expression of FoxM1 led to the loss expression of E-cadherin, which was significantly critical to the acquisition of EMT in non-small cell lung cancer (16). As alluded to previously, emerging clinical evidence shows that the downregulation of E-cadherin level is correlated with a poor prognosis of CRC via acquisition of an EMT phenotype (17). However, the underlying precise regulating mechanisms of E-cadherin remain unknown.

Based on the published literature, although a diverse array of studies can be found on FoxM1, Cav-1 and E-cadherin, respectively, there are no studies concerning the associations among these proteins in CRC. The aim of the present study was to determine the clinicopathological features of FoxM1, Cav-1 and E-cadherin in CRC, and to investigate the potential associations among these proteins.

\section{Materials and methods}

Human tissue samples. The expression of FoxM1, Cav-1 and E-cadherin in CRC was analyzed employing a human CRC and normal tissue microarray (US Biomax Inc., Rockville, MD, USA). The tissue microarray contained 30 samples of CRC, 8 samples of normal tissues, 2 samples of lung metastases derived from CRC, 5 samples of lymph node metastases derived from CRC and 3 samples of ovary metastases derived from CRC. Each sample had 2 cores from the same specimen. The specimens were obtained from 17 men and 13 women. The tumor stage (tumor-node-metastasis classification) for the CRC was stage II for 7 samples, stage III for 17 samples and stage IV for 6 samples (18). The differentiation for the CRC was poorly-differentiated for 1 specimen, moderately-differentiated for 18 specimens and well-differentiated for 8 specimens (the remaining 3 specimens were of mucinous carcinoma). The use of the tissue samples was approved by the Institutional Review Board of Shanghai Jiaotong University Affiliated First People's Hospital (Shanghai, China). The patients' clinical information for the tissue microarray, including gender, age, pathological diagnosis, clinical stage, histological grade and metastasis, was provided by US Biomax.

Tissue immunohistochemistry. Standard immunohistochemical procedures were applied using anti-FoxM1 rabbit polyclonal antibody (1:100 dilution; catalog no. sc-500; Santa Cruz Biotechnology Inc., Dallas, TX, USA), anti-Cav-1 mouse monoclonal antibody (1:200 dilution; catalog no. 610406; BD Biosciences, Franklin Lakes, NJ, USA) and anti-E-cadherin mouse monoclonal antibody (1:200 dilution; catalog no. 610182; BD Biosciences). First, the 4- $\mu \mathrm{m}$ slices were dried in an incubator at $60^{\circ} \mathrm{C}$, then dewaxed in xylene, washed twice for $10 \mathrm{~min}$ each and rehydrated using a gradient from $100,95,85,75$ and $50 \%$ ethanol to pure distilled water with 5-min washes, respectively. Antigen retrieval was performed by heating the slides dipped in $10 \mathrm{mM}$ sodium citrate $(\mathrm{pH} 6.0)$ at $95^{\circ} \mathrm{C}$ for $30 \mathrm{~min}$. Endogenous peroxidase activity was blocked by use of $3 \%$ hydrogen peroxide for $10 \mathrm{~min}$ at room temperature. The sections were incubated with $10 \%$ normal goat serum diluted with phosphate-buffered saline for $\sim 30$ min at room temperature. The slides were then incubated with the anti-FoxM1, anti-Cav-1 and anti-E-cadherin antibodies, respectively, at $4^{\circ} \mathrm{C}$ overnight. The specimens were later incubated with peroxidase-conjugated antibodies at normal room temperature for $1 \mathrm{~h}$. The secondary antibody used for FoxM1 was peroxidase-conjugated Affinipure goat antirabbit immunoglobulin $(\mathrm{Ig}) \mathrm{G}(\mathrm{H}+\mathrm{L})$ (1:500 dilution; catalog no. SA00001-2). The secondary antibody used for Cav-1 and E-cadherin was peroxidase-conjugated Affinipure goat anti-mouse $\mathrm{IgG}(\mathrm{H}+\mathrm{L})$ (1:500 dilution; catalog no. SA00001-1); All secondary antibodies were purified from antisera by immunoaffinity chromatography using antigens coupled to agarose beads. The slides were stained with diaminobenzidine and then counterstained with Mayer's hematoxylin. The tissue sections were dehydrated using 5-min washes with 50, 75, 85, 95 and $100 \%$ ethanol, respectively, and then washed twice with xylene for $10 \mathrm{~min}$ each. The slides were finally mounted with coverslips and assessed by microscopic examination (Axio Scope.A1; Zeiss AG, Oberkochen, Germany). 

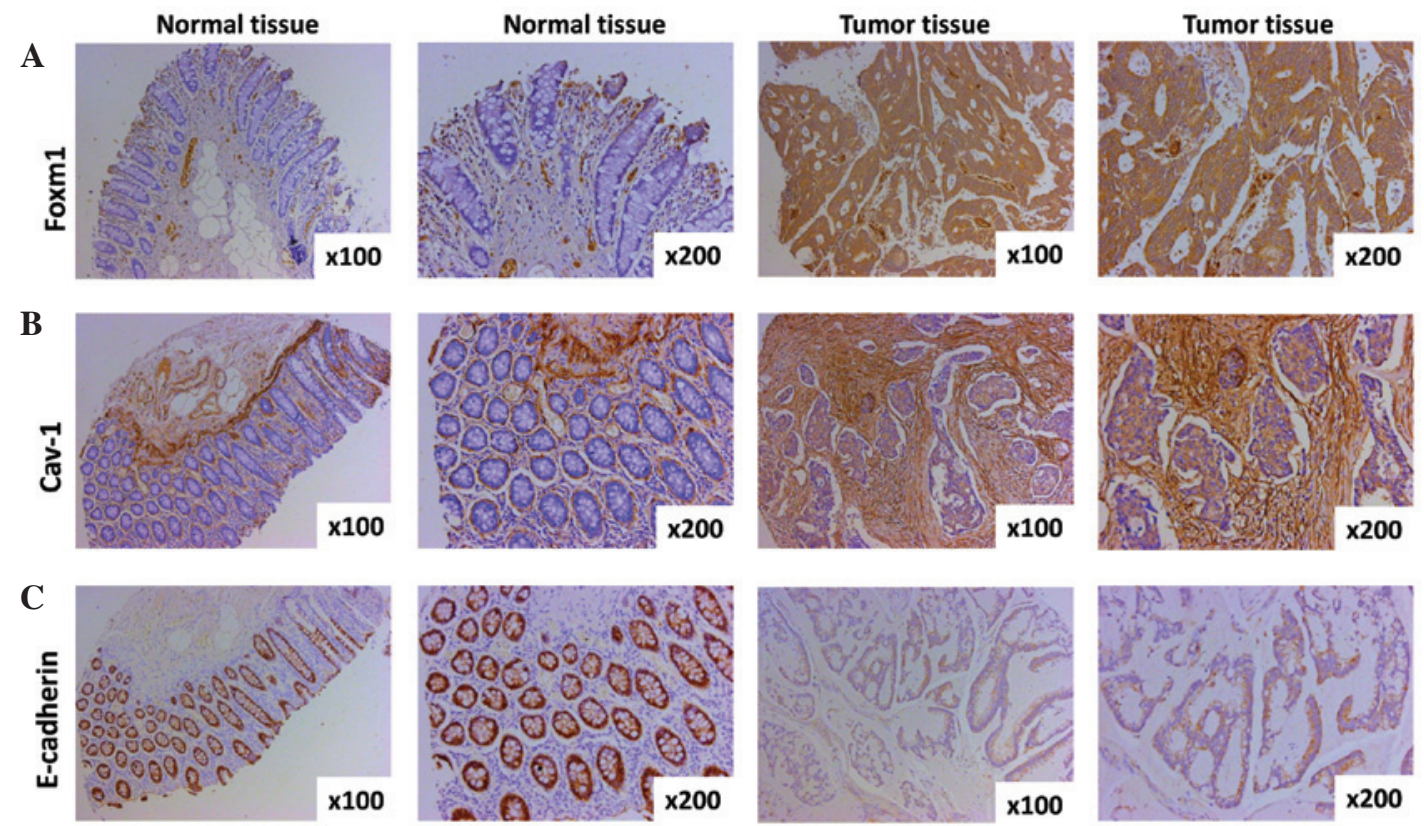

Figure 1. Expression of FoxM1, Cav-1 and E-cadherin in CRC specimens and normal tissues as detected by immunohistochemistry. (A) Representative images of FoxM1 protein expression in CRC tissues and surrounding normal tissues are shown (magnification, x100 and x200). The negative immunostaining of FoxM1 is displayed in the majority of the adjacent normal colorectal tissue cells, whereas the immunostaining of FoxM1 in the CRC tissues cells is strongly positive. (B) Cav-1 expression shown by immunohistochemical staining utilizing Cav-1 antibody (magnification, x100 and x200). The expression of Cav-1 in normal colorectal tissue cells is negative, however, the majority of areas of the CRC tissue cells are positive. (C) Typical images of E-cadherin protein expression in CRC tissues and paired normal tissue (magnification, x100 and x200). Almost all normal tissue cells are strongly positive, yet the majority of CRC cells are weakly positive or negative. CRC, colorectal cancer; FoxM1, Forkhead box protein M1; Cav-1, caveolin-1.

Immunohistochemistry score. Immunohistochemical scoring was implemented by two independent observers who were blinded to the clinical data. Depending completely on the proportion of positive cells and the intensity of cell staining in the field of view, the cell nuclei and cytoplasmic staining of FoxM1, the cytoplasmic and membranous staining of Cav-1, and the membranous staining of E-cadherin were relatively divided into three groups: Negative, weak and strongly positive. Collectively, the staining intensity of cells was classified into four intensity scores: No staining, 0; light staining, 1; moderate staining, 2; and dark staining, 3 . The percentage of positively-stained cells was classified into five grades with percentage scores as follows: $10 \%$ staining, 0 ; $10-25 \%$ staining, 1 ; 25-50\% staining, 2; 50-75 staining, 3; and $>75 \%$ staining, 4 . The staining positivity of FoxM1, Cav-1 and E-cadherin was calculated using the following formula: Total score $=$ intensity score $\mathrm{x}$ percentage score. Based on the total score of each tissue, results of $\leq 3,>3$ to $\leq 6$ and $>6$ were defined as negative, weakly-positive and strongly-positive, respectively.

Statistical analysis. The Wilcoxon rank sum test was performed to determine the significance of the association between the expression of FoxM1/Cav-1/E-cadherin and clinical parameters. The association between FoxM1 and Cav-1, FoxM1 and E-cadherin, and Cav-1 and E-cadherin protein expression was analyzed utilizing Spearman's test (r; P-value). In all tests, $\mathrm{P}<0.05$ was considered to indicate a statistically significant difference. SPSS 16.0 software (SPSS Inc., Chicago, IL, USA) was used for the statistical analyses.

\section{Results}

Expression of FoxM1, Cav-1 and E-cadherin in CRC tissues and surrounding normal tissues. The expression of FoxM1, Cav-1 and E-cadherin in CRC tissues and paired normal tissues was evaluated by immunohistochemistry. The expression of FoxM1 was observed mainly in the nuclear compartment and cytoplasm, and the staining of FoxM1 was weakly- or strongly-positive in $\mathrm{CRC}$ in comparison with negative in the adjacent normal tissue (Fig. 1A). A significant difference between CRC tissues and paired normal tissues was observed $\left(\mathrm{P}=8.5416 \times 10^{-6}\right) . \mathrm{Cav}-1$ expression was detected predominantly in the cytoplasm and cell membrane, and the extent of Cav-1 staining in the CRC tissues was weakly- or strongly-positive as compared with negative in the paired normal tissues $\left(\mathrm{P}=9.6306 \times 10^{-6}\right)$, suggesting that the expression of Cav-1 is upregulated in CRC (Fig. 1B). E-cadherin expression was primarily found in the membrane of epithelial cells, and staining for E-cadherin in the $\mathrm{CRC}$ and surrounding normal colorectal tissues was found to be negative (or weakly-positive) and strongly-positive, respectively (Fig. 1C). There was a significant difference in the expression of E-cadherin between the $\mathrm{CRC}$ tissues and paired normal tissues $(\mathrm{P}=0.0044)$.

Association between FoxM1 expression and clinicopathological features in CRC. The association between FoxM1 expression and the clinicopathological features in CRC was investigated using the immunohistochemistry tissues. The observations of the study supplied unequivocal evidence that the expression of FoxM1 was closely associated with tumor differentiation, tumor stage and its metastasis. A positive association was found between FoxM1 

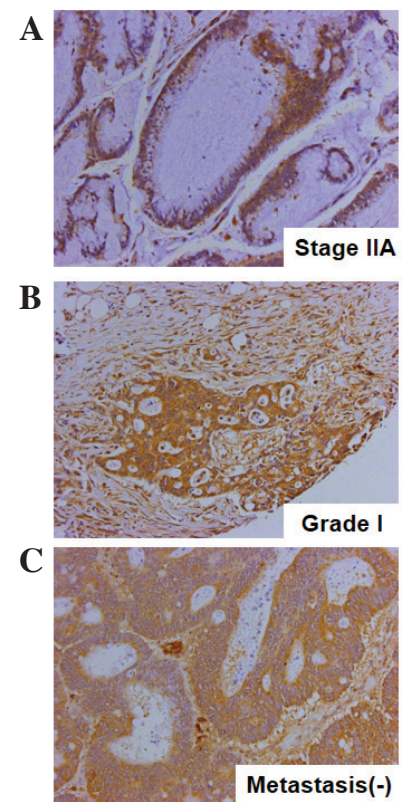
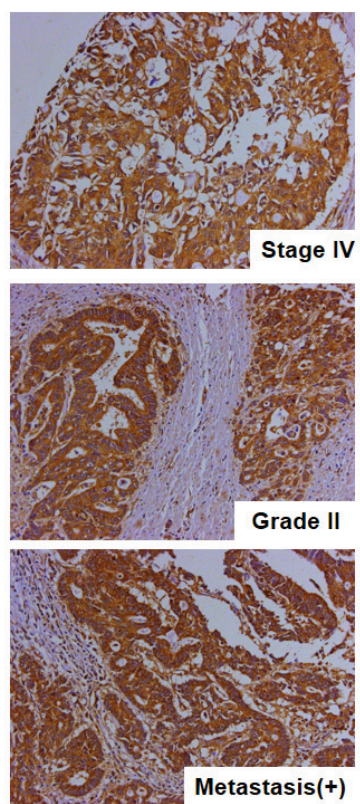
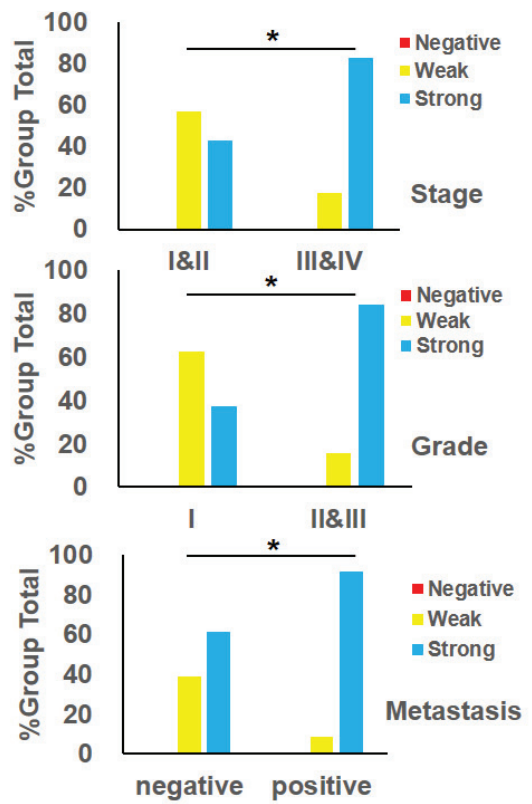

Figure 2. Association between the expression of FoxM1 and the clinicopathological features of colorectal cancer. (A) FoxM1 expression is positively associated with tumor stage (" $\mathrm{P}=0.0209$ for stages III and IV versus stages I and II); typical images of stage IIA and IV tumors are shown (magnification, x200). (B) FoxM1 expression is positively associated with tumor differentiation ( $\left({ }^{\mathrm{P}}=0.0355\right.$ for grade I versus grades II and III); typical images of grade I and II tumors are shown (magnification, $\mathrm{x} 200$ ). (C) FoxM1 expression is positively associated with tumor metastasis ( $\mathrm{P}=0.0038$ for the metastatic group versus the non-metastatic group); typical images of tumors with or without distant organ or lymph node metastasis are shown (magnification, x200). FoxM1, Forkhead box protein M1.

A

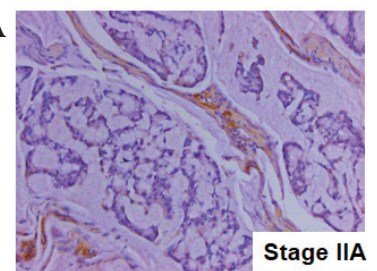

B

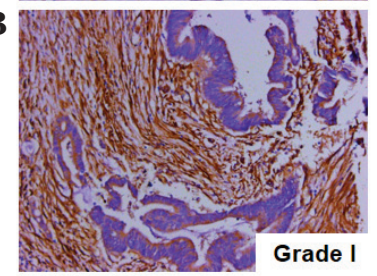

C

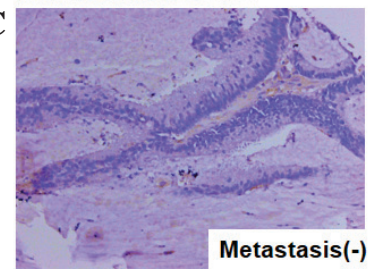

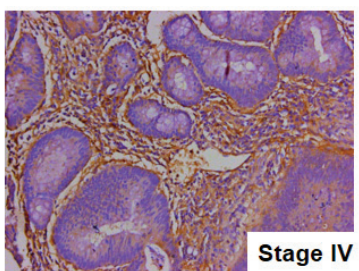

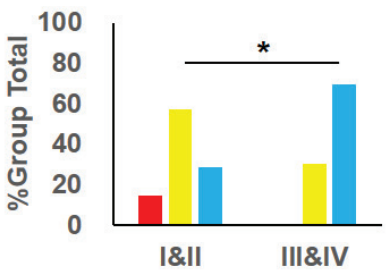

- Negative

Weak

- Strong

Stage
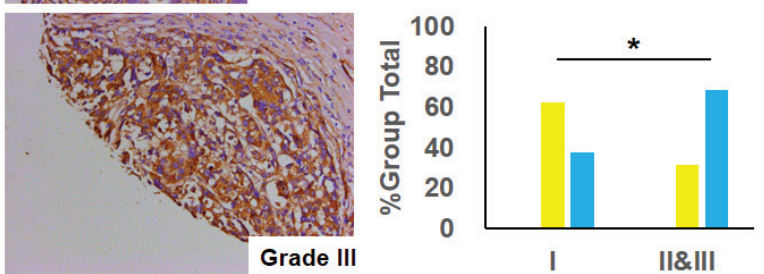

- Negative

Weak

- Strong

Grade
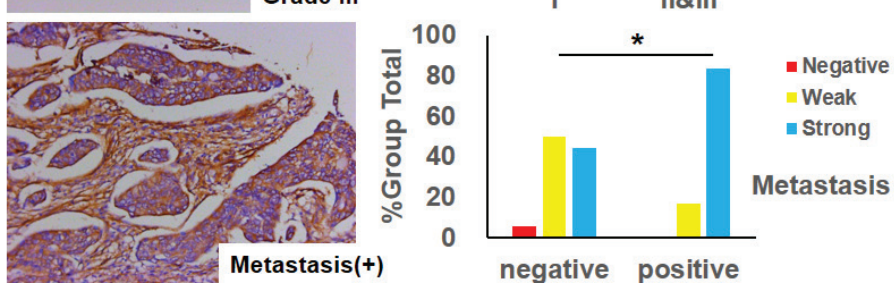

Figure 3. Cav-1 expression in colorectal cancer specimens and its association with the clinicopathological features. (A) Cav-1 expression is positively associated with tumor stage (" $\mathrm{P}=0.0477$ for stages III and IV versus stages I and II); typical images of stage IIA and IV tumors are shown (magnification, x200). (B) Cav-1 expression is positively associated with tumor differentiation ( $\mathrm{P}=0.0189$ for grade I versus grades II/III); typical images of grade I and III tumors are shown (magnification, $\mathrm{x} 200$ ). (C) Cav-1 expression is positively associated with tumor metastasis (" $\mathrm{P}=0.0112$ for the metastatic group versus the non-metastatic group); typical images of tumors with or without distant organ or lymph node metastasis are shown (magnification, x200). Cav-1, caveolin-1.

expression and tumor stage, indicating that the expression of FoxM1 was higher in advanced-stage tissues (stages III and IV) in contrast with early-stage tissues (stages I and II) (Fig. 2A). Additionally, when comparing well-differentiated (grade I), and moderately- and poorly-differentiated tumors (grades II and III), the FoxM1 expression was markedly increased in the moderately-differentiated tumors (Fig. 2B), and there was a significant difference between the two groups (grade I vs. grades II and III; $\mathrm{P}=0.0355)$. Furthermore, the expression of FoxM1 was much lower in non-metastatic samples than that in samples with distant organ or lymph node metastasis (Fig. 2C).

Association between Cav-1 expression and clinicopathological features in CRC. The status of Cav-1 expression was 

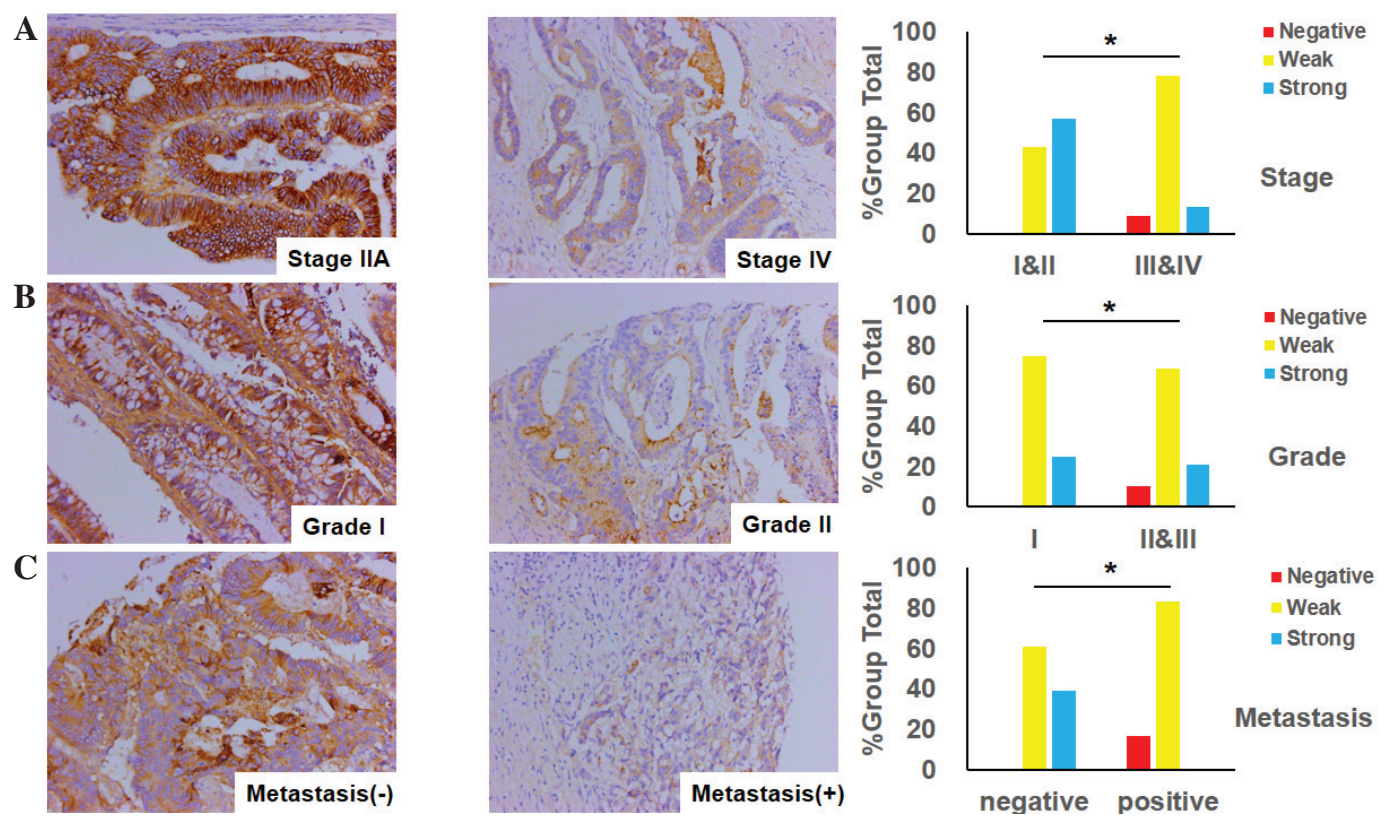

Figure 4. E-cadherin expression in colorectal cancer specimens and its association with the clinicopathological features. (A) E-cadherin expression is negatively associated with tumor stage ( $\mathrm{P}=0.0297$ for stages III and IV versus stages I and II); typical images of stage IIA and IV tumors are shown (magnification, $\mathrm{x} 200$ ). (B) E-cadherin expression is negatively associated with tumor differentiation ( $\mathrm{P}=0.0248$ for grade I versus grade II and III); typical images of grade I and II tumors are shown (magnification, $\mathrm{x} 200$ ). (C) E-cadherin expression is negatively associated with tumor metastasis ( $\mathrm{P}=0.0327$ for the metastatic group versus the non-metastatic group); typical images of tumors with or without distant organ or lymph node metastasis are shown (magnification, x200).

an important factor for CRC patients, as it was implicated in tumor differentiation and stage, and its metastasis. Fig. 3A shows that the level of Cav-1 expression in advanced-stage CRC (stages III and IV) was markedly positive, while conversely, the level of Cav-1 expression in early-stage CRC (stages I and II) was weak. There was a significant difference in Cav-1 expression between tumor stages $(\mathrm{P}=0.0477)$. In addition, it was identified that the expression of Cav-1 in poorly- and moderately-differentiated CRC tumors (grades II and III) was stronger compared with the weak expression of Cav-1 in well-differentiated tumors (grade I) (Fig. 3B). Moreover, it was found that the expression of Cav-1 in specimens of CRC with lymph node or distant organ metastasis was more elevated than that in other samples with no metastasis (Fig. 3C).

Association between the expression of the epithelial marker $E$-cadherin and clinicopathological features in CRC. The association between E-cadherin expression and clinicopathological variables is presented in Fig. 4. As illustrated in Fig. 4A, the expression of E-cadherin in advanced-stage CRC (stages III and IV) was observed to be more significantly decreased than in early-stage CRC (stages I and II). Similarly, the loss of expression of E-cadherin was more marked in the poorly- or moderately-differentiated tumors of CRC than in the well-differentiated tumors, as demonstrated in Fig. 4B. Consistent with the aforementioned observation, the expression of E-cadherin in CRC with lymph node or distant organ metastasis was attenuated more significantly than that in non-metastatic tissues (Fig. 4C).

Correlation among FoxM1, Cav-1 and E-cadherin expression. The strong or moderate staining of FoxM1 was observed in 30 out of 30 CRC tissues. Notably, among the 30 tissues strongly or moderately stained for FoxM1, there was 29 that were strongly or moderately stained for Cav-1. Similarly, the results showed that the expression of FoxM1 was strong or moderate in all the tissues stained strongly- or moderately for Cav-1 (29 out of 29). These observations suggested that FoxM1 expression in the CRC tissues had a positive correlation with Cav-1 expression ( $\mathrm{r}=0.569, \mathrm{P}=0.0103$ ) (Fig. 5A). With respect to the correlation between FoxM1 and E-cadherin expression, it was demonstrated that the increased expression of FoxM1 was always accompanied by the decreased expression of E-cadherin in the same CRC tissues Fig. 5A. On the other side, the elevated expression of E-cadherin was concomitant with the attenuated expression of FoxM1 in the same CRC tissues (Fig. 5B). It was clearly shown that a negative correlation existed between FoxM1 and E-cadherin expression ( $\mathrm{r}=-0.397$, $\mathrm{P}=0.0299$ ) (Fig. 5B). Consistent with these analogous observations, the increased expression of Cav-1 was observed together with the decreased expression of E-cadherin in corresponding CRC tissues. Additionally, Cav-1 expression was attenuated in contrast with the high E-cadherin expression in corresponding CRC specimens (Fig. 5C), indicating a negative association between Cav-1 and E-cadherin ( $\mathrm{r}=-0.362, \mathrm{P}=0.0492)$.

\section{Discussion}

The current study aimed to determine the critical associations among FoxM1, Cav-1 and E-cadherin in the development and progression of CRC. Based on the results, it was found that there was a positive correlation between FoxM1 and Cav-1 expression. Conversely, there was a negative correlation between FoxM1 and E-cadherin expression, and a negative correlation between Cav-1 and E-cadherin. This novel clinical evidence suggested that high FoxM1 expression, elevated 

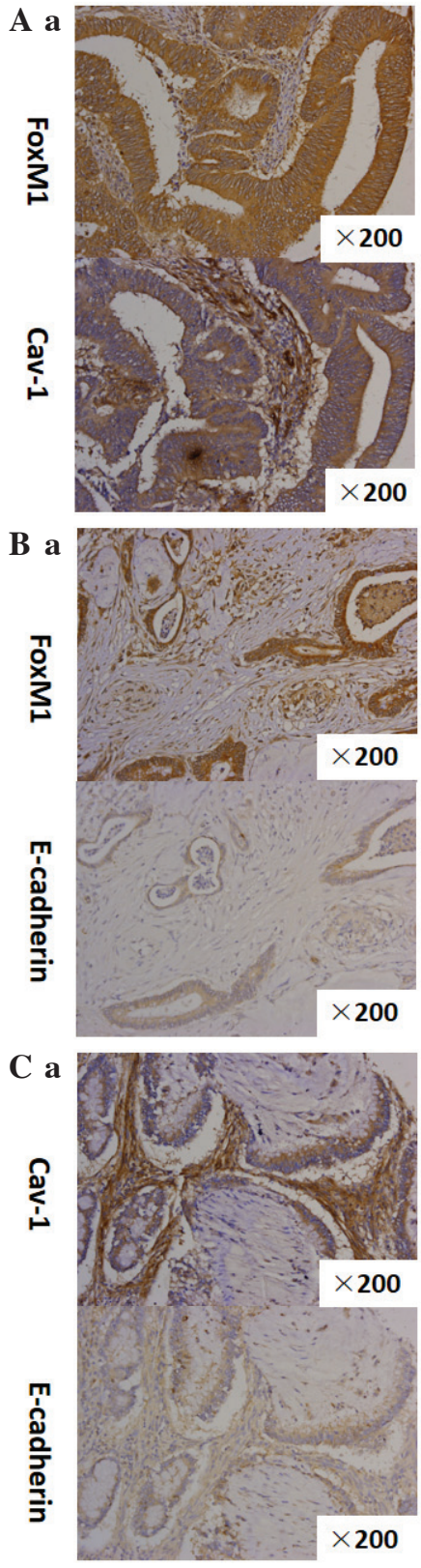

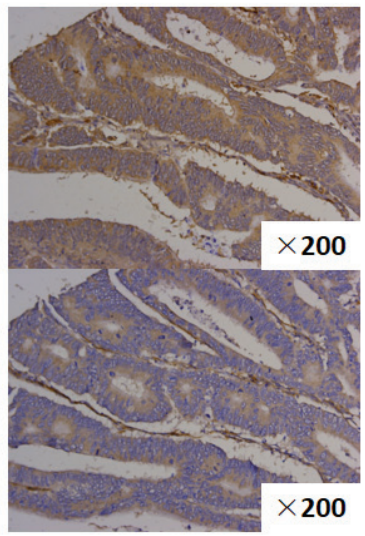

b
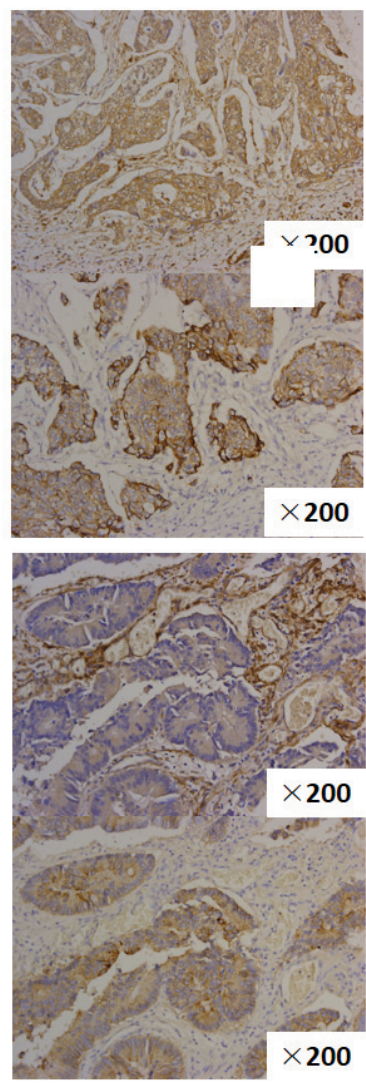

b

b
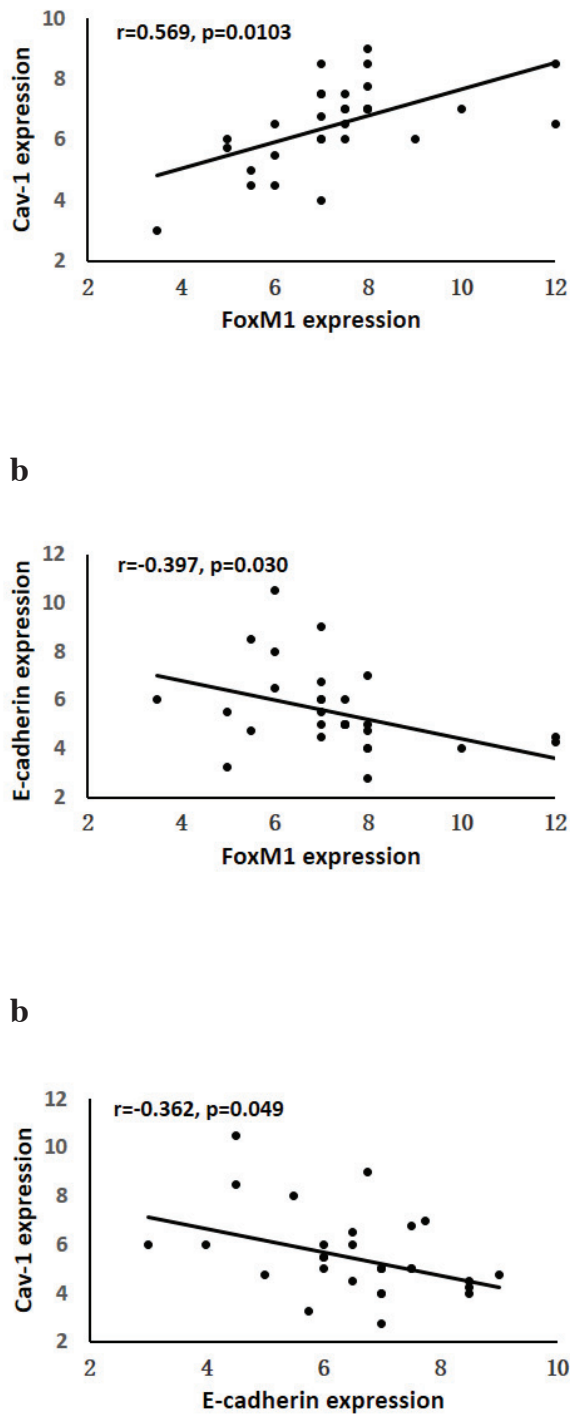

Figure 5. Correlation between FoxM1 and Cav-1 expression, FoxM1 and E-cadherin expression, and Cav-1 and E-cadherin expression. (Aa), FoxM1 and Cav-1 expression was detected by immunohistochemistry using antibodies for FoxM1 and Cav-1, respectively. Representative images of strongly-positive FoxM1 and Cav-1 staining (left 2 panels) and moderate FoxM1 and Cav-1 staining (right 2 panels) in CRC are shown (magnification, x200). (Ab) Direct correlation between FoxM1 and Cav-1 expression in CRC samples ( $\mathrm{n}=30$; Pearson's correlation test, $\mathrm{r}=0.533$ ). Certain dots on the graphs represent more than one sample (overlapped scores). (Ba) FoxM1 and E-cadherin expression were detected by immunohistochemistry using antibodies for FoxM1 and E-cadherin, respectively. Representative images of strongly-positive FoxM1 and weak E-cadherin staining (left 2 panels) and weakly-positive for FoxM1 and strongly-positive E-cadherin staining (right 2 panels) in CRC are shown (magnification, x200). (Bb), Direct corelation between FoxM1 expression and E-cadherin expression in CRC samples ( $n=30$; Pearson's correlation test, $r=-0.356$ ). Certain dots on the graphs represent more than one sample (overlapped scores). (Ca) Cav-1 and E-cadherin expression was detected by immunohistochemistry using antibodies for Cav-1 and E-cadherin, respectively. Representative images of strongly-positive Cav-1 and weakly-positive E-cadherin staining (left 2 panels) and weakly-positive Cav-1 and strongly-positive E-cadherin staining (right 2 panels) in CRC are shown (magnification, x200). (Cb) Direct correlation between Cav-1 and E-cadherin expression in CRC samples (n=30; Pearson's correlation test, $r=-0.342$ ). Certain dots on the graphs represent more than one sample (overlapped scores). CRC, colorectal cancer; FoxM1, Forkhead box protein M1; Cav-1, caveolin-1.

Cav-1 expression and low E-cadherin expression may be critical contributors to CRC pathogenesis and aggressiveness. Collectively, considering the evidence presented, a novel FoxM1/Cav-1/E-cadherin signaling pathway may exist in CRC, directly impacting EMT.

A growing body of evidence has convincingly demonstrated that FoxM1 plays an irreplaceable role in cancer initiation by modulating the $\mathrm{G}_{1}-\mathrm{S}$ and $\mathrm{G}_{2}-\mathrm{M}$ phases of the cell cycle, thus inducing cell proliferation and progression (19). Transcription molecules Skp2 and Cks1, components of the Skp-Cullin1-F-box complex, are controlled by FoxM1, which contributes greatly to the $\mathrm{G}_{1}-\mathrm{S}$ transition. Moreover, it is widely reported that FoxM1 is essential for the $\mathrm{G}_{2}-\mathrm{M}$ phase transition through regulators such as Cyclin A2, Cyclin B, 
survivin, centromere protein A and Aurora A kinase, which are under control of FoxM1 at the transcriptional level (20). It is accepted worldwide that FoxM1 is not only involved in cell proliferation, senescence and angiogenesis, which are indispensable for tumorigenesis, but that it is also implicated in EMT and metastasis, which are critical to tumor development and progression (20). Numerous clinical studies and experimental results have demonstrated that FoxM1, acknowledged as an oncogene, contributes to diseases such as Ewing's sarcoma, ovarian cancer, esophageal squamous cell carcinoma, gastric cancer and $\mathrm{CRC}$ by regulating the protein expression essential for angiogenesis, EMT and metastasis (7,21-24). One previous study provided convincing evidence that the increased expression of FoxM1 was clearly correlated with the poor prognosis of gastric cancer by downregulating E-cadherin expression in the cells of gastric cancer (21). In our previous study several lines of investigation were followed to determine the crucial role of FoxM1 in CRC, and the elevated expression of FoxM1 in in vivo and in intro experiments promoted the migration, invasion and metastasis of CRC. Conversely, the decreased expression of FoxM1 via the transfection of cells with small interfering RNA did the opposite (23). In the present study, it was demonstrated that the expression of FoxM1 was markedly associated with tumor stage $(\mathrm{P}=0.0209)$, grade $(\mathrm{P}=0.0355)$ and lymph node metastasis $(\mathrm{P}=0.0038)$. It was previously illustrated by Yang et al (25) that the overexpression of FoxM1 was accompanied by the decreased expression of E-cadherin in breast cancer, which was a pivotal step for the acquisition of an EMT phenotype in tumor invasion and metastasis. An unexpected finding in a study by Wierstra demonstrated that transcription factor FoxM1c can directly bind to the murine and human E-cadherin promoter as a novel target gene in tumors in vitro (26). In the present study, there was a negative correlation between FoxM1 and E-cadherin in CRC (Fig. 5B), indicating that FoxM1 may negatively regulate E-cadherin in the metastasis of CRC. Although the underlying mechanism of FoxM1 regulating the tumorigenicity and metastasis of cancer is unclear, all these observations support the fact that FoxM1 may be a valuable prognostic hallmark or serve as a novel therapeutic target in CRC.

It has been reported that Cav-1, also known as caveolin or VIP21, which was first identified in the caveolin family, plays an indispensable role in the physiological functions of the cell by functioning in surface signaling, intracellular cholesterol transport and endocytosis (27). Nevertheless, there is intense controversy concerning the role of Cav-1, either as a tumor suppressor or as a tumor promoter, in the process of cancer development and progression (10). One possible explanation accounting for the different roles of Cav-1 is the tumor type. A growing body of studies has shown that the decreased expression of Cav-1 can be recognized as a tumor suppressor in ovarian carcinoma (28), gastric cancer (11) and breast carcinoma (29), meanwhile, Cav-1 overexpression as a tumor promoter has been found in hepatocellular carcinoma (30), bladder cancer (31) and pancreatic cancer (15). Based on the available literature, the role of Cav-1 in CRC appears to be highly inconsistent. One study demonstrated that the overexpression of Cav-1 in colon cancer cells markedly weakened proliferation, development, invasion, viability and metastasis (32). Another study published by Patlolla et al (33) demonstrated that Cav-1 was overexpressed in the tissues and cell lines of human colon cancer. Besides that, emerging novel evidence has revealed that the expression of Cav-1 is a biphasic process in $\mathrm{CRC}$, meaning that the expression of $\mathrm{Cav}-1$ is commonly downregulated in early-stage CRC and then upregulated in advanced-stage CRC, manifesting the change in the role of Cav-1 in CRC from a oncogene to an anti-oncogene during tumor progression (34). In the present study, it was illustrated that the expression of Cav-1 was elevated in CRC tissues in comparison with paired normal tissues, and that the expression of Cav-1 was closely associated with tumor stage $(\mathrm{P}=0.0477)$, grade $(\mathrm{P}=0.0189)$ and lymph node metastasis $(\mathrm{P}=0.0112)$, which meant that it may act as a tumor promoter during the development of the tumor. Consistent with these observations, it was previously demonstrated that the elevated expression of Cav-1 in bladder cancer was tightly correlated with cell migration and invasiveness in contrast with the control group, and the expression of Cav-1 was elevated in the process of acquiring an EMT phenotype (31). Our previous study verified that the staining of Cav-1 in pancreatic cancer tissues was strongly positive compared with the paired normal tissues, and when Cav-1 expression was elevated in the pancreatic cancer cells, the abilities of cell migration and metastasis were strengthened, E-cadherin expression was significantly decreased and typical EMT morphological changes were caused (15). In the present study, it was found that the overexpression of Cav-1 in CRC was concomitant with the decreased expression of E-cadherin, which meant that Cav-1 may negatively regulate E-cadherin expression and play significant roles in the progression of CRC.

Depletion of E-cadherin, one of the predominant adhesive molecules of epithelial cells, plays a prominent role in the invasion and metastasis of diverse neoplasms, including bladder cancer, hepatocellular carcinoma and breast cancer, via acquisition of the EMT phenotype $(25,30,31)$. In the current study, it was shown that E-cadherin expression was decreased in CRC tissues, which is a key step in the process of acquiring an EMT phenotype, and the expression of E-cadherin was found to be closely associated with the clinicopathological features of the disease (Fig. 4). Diverse studies have revealed that there may be a range of regulatory mechanisms of E-cadherin in different cancers. In ovarian cancer, it was shown that transforming growth factor- $\beta$ played critical roles in tumorigenesis via decreasing E-cadherin expression (35). Moreover, it was demonstrated in non-small cell lung cancer that the expression of E-cadherin was regulated by microRNA-10b (36). However, the specific regulatory mechanism of E-cadherin in CRC remained unclear. Supporting data published by Yang et al (25) demonstrated that there was a correlation between FoxM1 and E-cadherin in breast cancer. Additionally, another study showed that Cav-1 can regulate the expression of E-cadherin in bladder cancer (31). In our previous study, it was verified that there was a novel FoxM1-Cav-1-E-cadherin signaling pathway in pancreatic cancer (15). Therefore, it may be concluded that a FoxM1-Cav-1-E-cadherin signaling pathway may exist in CRC.

Taken together, the results of the present study not only showed the expression of FoxM1, Cav-1 and E-cadherin in CRC tissues and normal tissues, but also revealed associations between FoxM1, Cav-1 and E-cadherin expression and 
clinicopathological features, respectively. More importantly, there may be a FoxM1-Cav-1-E-cadherin signaling pathway in CRC, which may be a promising therapeutic target for CRC patients.

\section{Acknowledgements}

The study was supported in part by grants from the Shanghai Municipal Human Resources and Social Security Bureau (nos. 2012040 and 13PJD024), the Shanghai Health and Family Planning Commission (no. XYQ2013092) and the Shanghai Municipal Science and Technology Commission (no. 14411966800).

\section{References}

1. Jemal A, Bray F, Center MM, Ferlay J, Ward E and Forman D: Global cancer statistics. CA Cancer J Clin 61: 69-90, 2011.

2. Chen W, Zheng R, Zhang S, Zhao P, Zeng H, Zou X and He J: Annual report on status of cancer in China, 2010. Chin J Cancer Res 26: 48-58, 2014.

3. Brenner H, Kloor M and Pox CP: Colorectal cancer. Lancet 383 : 1490-1502, 2014

4. Halasi M and Gartel AL: Targeting FOXM1 in cancer. Biochem Pharmacol 85: 644-652, 2013.

5. Alvarez-Fernandez M and Medema RH: Novel functions of FoxM1: From molecular mechanisms to cancer therapy. Front Oncol 3: 30, 2013.

6. Pilarsky C, Wenzig M, Specht T, Saeger HD and Grützmann R: Identification and validation of commonly overexpressed genes in solid tumors by comparison of microarray data. Neoplasia 6 : 744-750, 2004.

7. Christensen L, Joo J, Lee S, Wai D, Triche TJ and May WA: FOXM1 is an oncogenic mediator in Ewing Sarcoma. PloS One 8: e54556, 2013.

8. Yoshida Y, Wang IC, Yoder HM, Davidson NO and Costa RH: The forkhead box M1 transcription factor contributes to the development and growth of mouse colorectal cancer. Gastroenterology 132: 1420-1431, 2007.

9. Schwencke C, Braun-Dullaeus RC, Wunderlich $\mathrm{C}$ and Strasser RH: Caveolae and caveolin in transmembrane signaling: Implications for human disease. Cardiovasc Res 70: 42-49, 2006

10. Quest AF, Gutierrez-Pajares JL and Torres VA: Caveolin-1: An ambiguous partner in cell signalling and cancer. J Cell Mol Med 12: 1130-1150, 2008.

11. Kannan A, Krishnan A, Ali M, Subramaniam S, Halagowder D and Sivasithamparam ND: Caveolin-1 promotes gastric cancer progression by up-regulating epithelial to mesenchymal transition by crosstalk of signalling mechanisms under hypoxic condition. Eur J Cancer 50: 204-215, 2014.

12. Bender FC, Reymond MA, Bron C and Quest AF: Caveolin-1 levels are down-regulated in human colon tumors, and ectopic expression of caveolin-1 in colon carcinoma cell lines reduces cell tumorigenicity. Cancer Res 60: 5870-5878, 2000.

13. Fine SW, Lisanti MP, Galbiati F and Li M: Elevated expression of caveolin-1 in adenocarcinoma of the colon. Am J Clin Pathol 115 719-724, 2001.

14. Gumbiner BM: Regulation of cadherin-mediated adhesion in morphogenesis. Nat Rev Mol Cell Biol 6: 622-634, 2005.

15. Huang C, Qiu Z, Wang L, Peng Z, Jia Z, Logsdon CD, Le X, Wei D, Huang S and Xie K: A novel FoxM1-caveolin signaling pathway promotes pancreatic cancer invasion and metastasis. Cancer Res 72: 655-665, 2012.

16. Kong FF, Qu ZQ, Yuan HH, Wang JY, Zhao M, Guo YH, Shi J, Gong XD, Zhu YL, Liu F, et al: Overexpression of FOXM1 is associated with EMT and is a predictor of poor prognosis in non-small cell lung cancer. Oncol Rep 31: 2660-2668, 2014.
17. He X, Chen Z, Jia M and Zhao X: Downregulated E-cadherin expression indicates worse prognosis in Asian patients with colorectal cancer: Evidence from meta-analysis. PloS One 8: e70858, 2013

18. Edge S, Byrd DR, Compton CC, Fritz AG, Greene FL and Trotti A (eds): AJCC Cancer Staging Manual. 7th edition. Springer, NY, 2010.

19. Huang C, Du J and Xie K: FOXM1 and its oncogenic signaling in pancreatic cancer pathogenesis. Biochim Biophys Acta 1845: 104-116, 2014.

20. Lam EWF, Brosens JJ, Gomes AR and Koo CY: Forkhead box proteins: Tuning forks for transcriptional harmony. Nat Rev Cancer 13: 482-495, 2013.

21. Miao L, Xiong X, Lin Y, Cheng Y, Lu J, Zhang J and Cheng N: Down-regulation of FoxM1 leads to the inhibition of the epithelial-mesenchymal transition in gastric cancer cells. Cancer Genet 207: 75-82, 2014.

22. Takata A, Takiguchi S, Okada K, Takahashi T, Kurokawa Y, Yamasaki M, Miyata H, Nakajima K, Mori M and Doki Y: Clinicopathological and prognostic significance of FOXM1 expression in esophageal squamous cell carcinoma. Anticancer Res 34: 2427-2432, 2014.

23. Li D, Wei P, Peng Z, Huang C, Tang H, Jia Z, Cui J, Le X, Huang S and Xie K: The critical role of dysregulated FOXM1-PLAUR signaling in human colon cancer progression and metastasis. Clin Cancer Res 19: 62-72, 2013.

24. Huang C, Xie D, Cui J, Li Q, Gao Y and Xie K: FOXM1c promotes pancreatic cancer epithelial-to-mesenchymal transition and metastasis via upregulation of expression of the urokinase plasminogen activator system. Clin Cancer Res 20: 1477-1488, 2014.

25. Yang C, Chen H, Tan G, Gao W, Cheng L, Jiang X, Yu L and Tan Y: FOXM1 promotes the epithelial to mesenchymal transition by stimulating the transcription of Slug in human breast cancer. Cancer Lett 340: 104-112, 2013.

26. Wierstra I: The transcription factor FOXM1c binds to and transactivates the promoter of the tumor suppressor gene E-cadherin. Cell Cycle 10: 760-766, 2011

27. Williams TM and Lisanti MP: The caveolin proteins. Genome Biol 5: 214, 2004.

28. Sanna E, Miotti S, Mazzi M, De Santis G, Canevari S and Tomassetti A: Binding of nuclear caveolin-1 to promoter elements of growth-associated genes in ovarian carcinoma cells. Exp Cell Res 313: 1307-1317, 2007.

29. Martins D, Beça FF, Sousa B, Baltazar F, Paredes J and Schmitt F: Loss of caveolin-1 and gain of MCT4 expression in the tumor stroma: Key events in the progression from an in situ to an invasive breast carcinoma. Cell Cycle 12: 2684-2690, 2013.

30. Müller R, Gai X, Lu Z, Tu K, Liang Z and Zheng X: Caveolin-1 Is up-regulated by GLI1 and contributes to GLI1-driven EMT in hepatocellular carcinoma. PloS one 9: e84551, 2014.

31. Liang W, Hao Z, Han JL, Zhu DJ, Jin ZF and Xie WL: CAV-1 contributes to bladder cancer progression by inducing epithelial-to-mesenchymal transition. Urologic Oncol 32: 855-863, 2014

32. Nimri L, Barak H, Graeve L and Schwartz B: Restoration of caveolin-1 expression suppresses growth, membrane-type-4 metalloproteinase expression and metastasis-associated activities in colon cancer cells. Mol Carcinog 52: 859-870, 2013.

33. Patlolla JM, Swamy MV, Raju J and Rao CV: Overexpression of caveolin-1 in experimental colon adenocarcinomas and human colon cancer cell lines. Oncol Rep 11: 957-963, 2004.

34. Ha TK, Her NG, Lee MG, Ryu BK, Lee JH, Han J, Jeong SI, Kang MJ, Kim NH, Kim HJ, et al: Caveolin-1 increases aerobic glycolysis in colorectal cancers by stimulating HMGA1-mediated GLUT3 transcription. Cancer Res 72: 4097-4109, 2012.

35. Gao J, Zhu Y, Nilsson M and Sundfeldt K: TGF-b isoforms induce EMT independent migration of ovarian cancer cells. Cancer Cell Int 14: 72, 2014.

36. Zhang J, Xu L, Yang Z, Lu H, Hu D, Li W, Zhang Z, Liu B and Ma S: MicroRNA-10b indicates a poor prognosis of non-small cell lung cancer and targets E-cadherin. Clin Transl Oncol 17: 209-214, 2015. 